\title{
Short communication: Genetic association of variations in the osteopontin gene (SPP1) with lactation persistency in dairy cattle
}

\section{Nathalie Bissonnette ${ }^{1}$}

Sherbrooke Research and Development Centre, Agriculture and Agri-Food Canada, 2000 College Street, Sherbrooke, Quebec, J1M 0C8 Canada

\begin{abstract}
Improving lactation persistency (LP) in dairy cattle has a beneficial effect on animal health and fertility and herd productivity. A complex trait, LP not only reflects the cow's ability to maintain milk secretion activity after the lactation peak but is also a function of the postcalving development of the mammary gland and, later on, of tissue remodeling as lactation declines. This decline is a consequence of an imbalance between cell proliferation and cell removal. In a previous study, single nucleotide polymorphisms were identified in the osteopontin (OPN) gene, SPP1. Osteopontin is a multifaceted protein that plays an important role in immune regulation and tissue remodeling. Because OPN is involved in involution, it might also have an effect on LP. The objective of the present study was to evaluate whether LP could be influenced by genetic variations in the SPP1 gene. This association with LP was analyzed in the population of 578 bulls characterized in a previous study. The population mean of estimated breeding value $(\mathrm{EBV})$ for $\mathrm{LP}$ was $100.95 \pm 5.06$ units. Allele and genotype association analyses were performed by comparing the frequencies of the different genotypes and alleles with EBV for LP for the respective lactation using logistic regression. The EBV for LP at the first lactation (LP1), second lactation (LP2), and third lactation (LP3) and for overall lactation (OLP) are reported for the genotypes $S P P 1 c .-1301 G>A, S P P 1 c .-1251 C>T$, $S P P 1 c .-430 G>A$, and $S P P 1 c .{ }^{*} 40 A>C$. The first single nucleotide polymorphism, $S P P 1 c .-1301 G>A$, affected LP1, LP2, LP3, and OLP. Analysis of the estimated average allele substitution effects also confirmed that $\mathrm{G}$ is a favorable allele for LP, given the gain observed over LP1, LP2, LP3, and OLP. Differences in EBV for LP were observed between animals with different haplotypes at LP1, LP2, LP3, and OLP. Contrast analysis for OLP revealed that mean EBV is greater for block H1 $(101.34 \pm 0.30)$ than for animals that do not have
\end{abstract}

Received May 8, 2017.

Accepted September 23, 2017.

${ }^{1}$ Corresponding author: nathalie.bissonnette@agr.gc.ca
H1 $(98.20 \pm 0.77)$. The gain with block H1 (GCGA) suggests the presence of the favorable allele $G$ (first position in the block: SPP1c.-1301G). The pleiotropic roles of OPN position it at the crossroads of immune regulation, tissue remodeling, and involution. From a genetic perspective, data from the present study suggest OPN as a candidate gene associated with LP for dairy cows.

Key words: lactation persistency, osteopontin, genetic association, dairy bovine

\section{Short Communication}

In dairy cattle, extended lactations have a beneficial effect on animal health and fertility and herd productivity. The lactation persistency ( $\mathbf{L P})$ trait is considered part of a strategy to decrease stressful peak yields associated with health problems while maintaining a high level of production after peak yield. The heritability of LP is moderate (0.18; Boettcher, 2005; Cole and VanRaden, 2006) to substantial (0.34, first lactation; Jamrozik et al., 2000). The EBV for LP, which have been available since 2000 in Canadian Holstein, is calculated for the first 3 lactations (Canadian Dairy Network, 2004). The LP can be computed based on solutions from the random regression test-day model (Jamrozik et al., 1998).

Few studies have investigated relationships between LP and health performance. Disease traits such as mastitis or SCS were found to confirm that LP deserves consideration in a breeding program (Cole and VanRaden, 2006; Appuhamy et al., 2007). Other health problems such as metabolic stress and negative energy balance were found to be negatively correlated with LP (Jakobsen et al., 2003; Walsh et al., 2011). On the whole, however, studies on extended lactation performance are scarce because LP is a complex trait. Lactation persistency not only reflects the ability to maintain milk secretion activity after the lactation peak but is also a function of postcalving mammary gland development and, later on, of tissue remodeling as lactation declines. This decline in milk yield after peak lactation in dairy animals reflects a decrease in the number of 
mammary cells, resulting from an imbalance between cell proliferation and cell removal (Stefanon et al., 2002; Capuco et al., 2003). Interestingly, in a genome-wide association study on LP, gene-enriched QTL showed that several networks related to cell development, proliferation, and death were enriched for LP positional candidate genes (Do et al., 2017). Numerous factors influence LP, notably hormonal changes (Stefanon et al., 2002; Yart et al., 2013, 2014) and cell regulation mechanisms leading to apoptosis (Sorensen et al., 2006; Norgaard et al., 2008). During involution, remodeling of mammary tissue occurs (Herve et al., 2016). This process is characterized by extensive cell death and the acquisition of an inflammatory profile. These events have been described as the signature of wound healing, including an influx of macrophages that support tissue remodeling (O'Brien et al., 2012).

The protein osteopontin ( OPN) is secreted in numerous body fluids, including blood and milk. This multifaceted protein plays an important role in tissue homeostasis and remodeling, immune regulation, and stress response (Denhardt and Noda, 1998; Denhardt et al., 2001; Sodek et al., 2006; Wang and Denhardt, 2008), and also contributes to macrophage homing and cellular immunity (Ashkar et al., 2000). Expression of the OPN gene (SPP1) increases locally in the bovine mammary gland during bacterial infection to improve cell-mediated immunity (Denhardt et al., 2001; Konno et al., 2006; Alain et al., 2009). Osteopontin is naturally present in bovine milk, and interestingly, genetic variations of a cow SSP1 influence the amount of OPN secreted in its milk throughout lactation (Dudemaine et al., 2014). The gene SPP1 is part of the molecular mechanisms involved during involution of the mammary gland in mice (Baik et al., 1998; Prokesch et al., 2014). The hypothesis of the present study is that OPN may also affect LP because of its role in involution.

In a previous study, $4 \mathrm{SNP}$ in $S P P 1$ were identified to be associated with the mammary health status of dairy cows (Alain et al., 2009). These SNP, namely, $S P P 1 c .-1301 G>A \quad($ rs109637038), $\quad S P P 1 c .-1251 C>T$ (rs109637038), SPP1c.-430G>A (rs108997065), and $S P P 1 c . * 40 A>C \quad(\operatorname{rs} 132812135)$, were identified by sequencing the promoter, exons, and $3^{\prime}$ untranslated region of animals with extreme EBV for SCS (Alain et al., 2009). From these SNP, the haplotype blocks H1 to H5 were defined. The analysis of the functional effect of the haplotypes confirmed that genetic variations in the SPP1 promoter affect the level of OPN secretion into bovine milk (Dudemaine et al., 2014). In addition, immune cells isolated from cows with different haplotypes differentially respond to bacterial ex vivo infection. As these SNP affect gene expression, they may account
Table 1. Genotypes and haplotype frequencies of the 578 bull population

\begin{tabular}{lccccc}
\hline \multicolumn{3}{c}{ Genotype $^{1}$} & & \\
\cline { 1 - 2 } & Allele 1 & & Allele 2 & Frequency & $\%$ \\
\hline H1 & (GCGA) & H1 & (GCGA) & 205 & 35.5 \\
H1 & (GCGA) & H2 & (GTAC) & 170 & 29.4 \\
H1 & (GCGA) & H3 & (ACGA) & 76 & 13.1 \\
H1 & (GCGA) & H4 & (GCGC) & 30 & 5.2 \\
H2 & (GTAC) & H2 & (GTAC) & 37 & 6.4 \\
H2 & (GTAC) & H3 & (ACGA) & 28 & 4.8 \\
H2 & (GTAC) & H4 & (GCGC) & 13 & 2.2 \\
H3 & (ACGA) & H3 & (ACGA) & 6 & 1.0 \\
H3 & (ACGA) & H4 & (GCGC) & 5 & 0.9 \\
H4 & (GCGC) & H4 & (GCGC) & 2 & 0.3 \\
H4 & (GCGC) & H5 & (GTAA) & 6 & 1.0 \\
Total & & & & 578 & 100 \\
\hline
\end{tabular}

${ }^{1} \mathrm{H} 1-\mathrm{H} 4=$ haplotype blocks 1 to 4.

for phenotypic variation of milk production and health traits (Dudemaine et al., 2014). The objective of the present study was to evaluate whether LP could be influenced by genetic variations in the SPP1 gene. The association of these SNP with LP was therefore analyzed in the population of 578 bulls characterized in a previous study (Alain et al., 2009). The genotypes and haplotype frequencies of the 4 SNP identified among the 578 bulls are summarized in Table 1.

For the Holsteins breed, bull proofs for LP reflect the average daughter milk yield at lactation d 280 compared with lactation d 60 . The scale used is standardized so that, in the base population, the calculated weighted percentage of EBV for LP has a mean of 100 and a standard deviation of 5 . As a result, LP is an arbitrary relative value with no defined units. The EBV of LP for the 578 bulls were provided by the Canadian Dairy Network (Guelph, ON, Canada; https://www.cdn.ca/). The mean for the EBV of LP of the 578 bulls was $100.95 \pm 5.06$ units. Allele and genotype association analyses were performed by comparing the frequencies of the different genotypes and alleles with EBV for LP for the respective lactation. Data were analyzed using the SAS software (release 9.4, SAS Institute Inc., Cary, NC), The model for either genotype or haplotype effect was a one-way factorial with unequal variances due to the strongly unbalanced number of animals per group. When a genotype effect was found significant, a Tukey adjusted multiple comparisons was further performed. The EBV for LP at the first lactation (LP1), second lactation (LP2), and third lactation (LP3) and for overall lactation (OLP) are reported in Table 2 for genotypes SPP1c.-1301G>A, SPP1c.-1251C>T, $S P P 1 c .-430 G>A$, and $S P P 1 c .{ }^{*} 40 A>C$. The first SNP, $S P P 1 c .-1301 G>A$, affected LP1, LP2, LP3, and OLP $(P<0.001)$. The estimated haplotype frequencies of 
Table 2. Effects of SNP in the bovine osteopontin gene (SPP1) on EBV for lactation persistency for overall lactation (OLP) and for the first lactation (LP1), second lactation (LP2), and third lactation (LP3) ${ }^{1}$

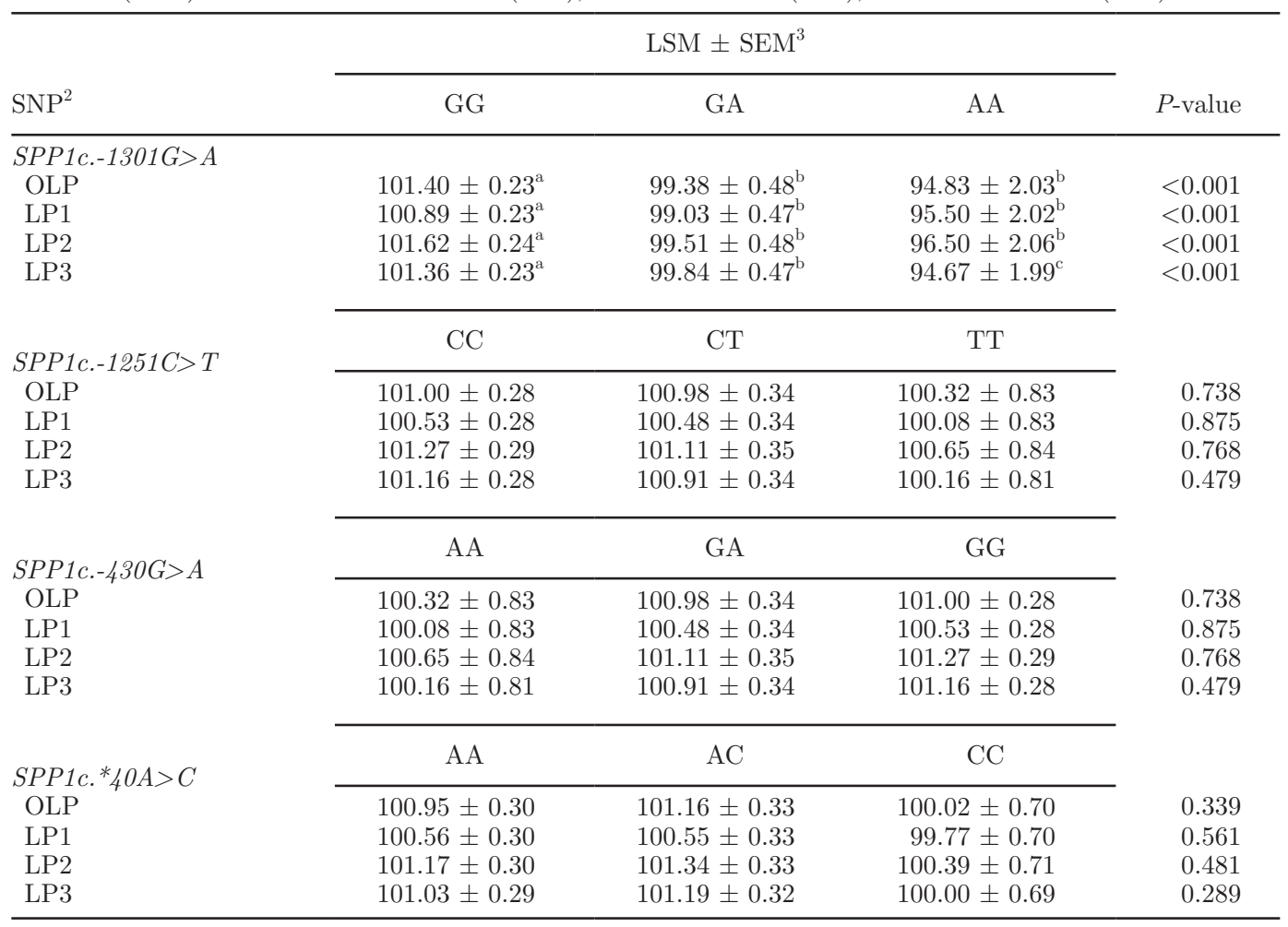

${ }^{\mathrm{a}-\mathrm{c}}$ Means within a row with different superscripts differ $(P<0.05)$.

${ }^{1}$ The 3 individual lactation proofs using EBV for persistency are combined to produce the overall lactation persistency (OLP): the emphasis, $50 \%$ of EBV, is placed on the first lactation (LP1), whereas the second lactation persistency (LP2) and third lactation persistency (LP3) each receive a weight of $25 \%$ (Canadian Dairy Network, 2004).

${ }^{2} S P P 1 c .-1301 G>A$ refers to rs109637038, SPP1c.-1251C>T refers to rs109637038, SPP1c. $-430 G>A$ refers to rs108997065, and $S P P 1 c .{ }^{*} 40 A>C$ refers to rs132812135.

${ }^{3}$ The LSM are the adjusted means of the EBV for lactation persistency for bulls, which are grouped by genotype for each SNP and calculated for the respective lactation.

the 578 bulls showed previously that 5 haplotypes (H1 to $\mathrm{H} 5)$ represent $99.2 \%$ of the alleles in this population (Alain et al., 2009). Differences were also observed between haplotype groups with EBV for LP at LP1 $(P$ $=0.001), \mathrm{LP} 2(P=0.001), \mathrm{LP} 3(P=0.002)$, and OLP $(P<0.001$; Table 3$)$.

The average allele substitution effects of the SNP were calculated using the following model also described in Leyva-Baca et al. (2008):

$$
Y_{j k}=\mu+\sum_{i=1}^{4} \beta_{i} G_{i}+S_{j}+e_{j k}
$$

where $y_{j k}=$ trait EBV for the $j$ th bull from the $k$ th sire, $\mu=$ overall mean, $\beta_{i}=$ fixed regression coefficient for the $i$ th SNP, $G_{i}=$ the genotype of the $i$ th SNP recoded, as described elsewhere (Zeng et al., 2005), as follows:

$$
G_{i}=\left\{\begin{array}{c}
-1 \text { for homozygous genotype } 1 \\
0 \text { for heterozygous } \\
+1 \text { for homozygous genotype } 2
\end{array},\right.
$$

$S_{j}=$ random polygenic effect of the $j$ th sire $(j=1$ to $26)$, and $e_{j k}=$ random error.

This analysis confirmed that $\mathrm{G}$ is a favorable allele for LP, given the gain observed over LP1 $(P=0.007)$, LP2 $(P=0.013)$, LP3 $(P=0.050)$, and OLP $(P=0.009$; Table 4). No deregression of the EBV for LP with the parent average removed was applied to generate higher reliabilities of genomic breeding values. It might have improved haplotype substitution effects, which was not found to be significant (data not shown). However, the difference was significant at a comparison-wise level with EBV for LP. Indeed, contrast analysis for OLP 
revealed that mean EBV is greater $(P<0.001)$ for block H1 $(101.34 \pm 0.30)$ than for animals that do not have H1 (98.20 \pm 0.77$)$. The $1.98 \%$ gain with block H1 (GCGA) suggests a positive effect on the presence of allele G (first position in the block: allele SPP1c.-1301G). In a previous study (Dudemaine et al., 2014), OPN secretion was quantified in milk throughout lactation for 2 groups of cows harboring haplotypes H1-GCGA $\times$ H4-GCGC $(\mathrm{n}=5)$ and H2-GTAC $\times$ H3-ACGA $(\mathrm{n}$ $=5$ ). Marked increases in both concentration and total quantity of OPN were found for $\mathrm{H} 1 \times \mathrm{H} 4$ cows in comparison with $\mathrm{H} 2 \times \mathrm{H} 3$ cows starting in wk 25 of lactation (Dudemaine et al., 2014). The H1 and H4 haplotypes contain the same promoter segment (the SNP GCG). Using functional analysis of the promoter of $S P P 1$, it was shown that the activity of the segment H1-GC was greater $(P<0.001)$ than the activity of H2 and H3 (Dudemaine et al., 2014). Interestingly, in the present study, contrast analysis performed for LP for these haplotype groups (i.e., $\mathrm{H} 1 \times \mathrm{H} 4$ vs. $\mathrm{H} 2 \times$ H3) also confirmed a difference for LP1 $(P=0.012)$, LP2 $(P<0.001)$, LP3 $(P<0.001)$, and OLP $(P<$ $0.001)$. However, this gain of $1.98 \%$ for OLP obviously cannot be attributed only to SPP1c.-1301G because other haplotypes (i.e., H2, H4, and H5) also carry this genotype without having a similar beneficial effect on LP. Considering that the 4 SNP from this analysis were identified in a population selected based on extreme EBV for SCC, other genetic variations could have been identified for the LP trait using bulls having extreme EBV for LP. Thus, sequencing the exons, introns, and promoter region of those bulls having extreme EBV for LP would enable identification of additional SNP with SPP1c.-1301G and provide a better understanding of the molecular map of genetic variations associated with LP. Notwithstanding the missing pieces of the puzzle, a gain of $\sim 2 \%$ of LP is an important economic benefit that can be attributed to numerous factors, including milk production yields, metabolic disease, and reproductive performance. Higher 305-d yields and greater LP were associated with a longer calving interval (Muir et al., 2004); together, these factors have a positive effect on longevity (Walker, 2014; Nayeri et al., 2017). The numerous factors affecting LP make it difficult to identify the source of the $\sim 2 \%$ gain and therefore to quantify the economic gain.

From a molecular perspective, variation at the position of the SNP SPP1c.-1301G>A can potentially affect 2 recognition motifs: the mammalian transcrip-

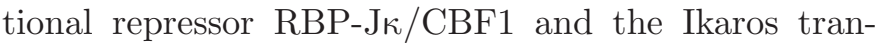
scription factor (IKZF1/2). In silico analysis of both alleles using the MatInspector search tool from the Genomatix software suite (www.genomatix.de) suggests that SPP1c.-1301G abolishes both the RBP-Jк/ 
Table 4. Estimated genotype effects on EBV for lactation persistency for overall lactation (OLP) and for the first lactation (LP1), second lactation (LP2), and third lactation (LP3) ${ }^{1}$

Average genotype substitution effect in EBV units

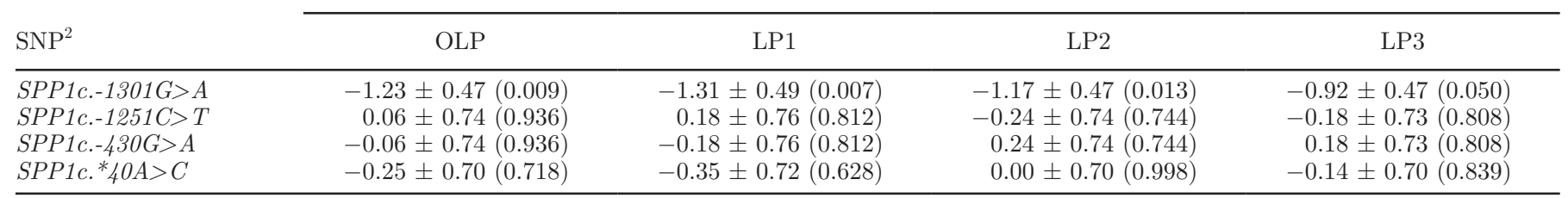

${ }^{1}$ The 3individual lactation proofs using EBV for persistency are combined to produce the overall lactation persistency (OLP): the emphasis, $50 \%$ of EBV, is placed on the first lactation (LP1), whereas the second lactation persistency (LP2) and third lactation persistency (LP3) each receive a weight of 25\% (Canadian Dairy Network, 2004). Values are presented as estimated genotype effect \pm SEM $(P$-value).

${ }^{2} S P P 1 c .-1301 G>A$ refers to rs109637038, SPP1c. $-1251 C>T$ refers to rs109637038, SPP1c. $-430 G>A$ refers to rs108997065, and $S P P 1 c . * 40 A>C$ refers to rs132812135.

CBF1 and IKZF1/2 binding motifs but SPP1c.-1301A does not (data not shown). The motif IKZF1/2 was also identified in a previous study (Alain et al., 2009) as being associated with the allele SPP1c.-1301A, using the software MOTIF Search (http://motif.genome.jp/). Ikaros appears to exert its influence on chromatin remodeling through the induction of epigenetic changes that are characteristic of transcriptionally repressed chromatin (Gowda et al., 2016; Wang et al., 2016). Interestingly, the second DNA-binding factor, RBP-J, is a methyl-CpG-binding protein (Bartels et al., 2011) and thus also has the capacity to repress the activity of chromatin through epigenetic modifications (Schwanbeck, 2015). Moreover, RBP-J was previously found to switch off target gene expression and ensure the tight spatiotemporal control of the gene expression response of the target gene (Liefke et al., 2010). Although the interaction of Ikaros or RBP-J with the promoter of $S P P 1$ has not been confirmed, the genetic analysis deserves consideration because of the $S P P 1 c .-1301 G>A$ functional marker. From a genetic perspective, it is well known that health and disease may affect other performance traits such as longevity and LP. Osteopontin is a multifaceted protein at the crossroads of immune regulation, wound healing, tissue remodeling, and involution. Data from the present study suggest OPN as a candidate gene associated with LP for dairy cows.

\section{ACKNOWLEDGMENTS}

Statistical analyses were performed by S. Méthot (Agriculture and Agri-Food Canada, Sherbrooke, Canada). The author thanks Semex Alliance Inc. (SaintHyacinthe, Canada) for providing semen samples and Filippo Miglior (Canadian Dairy Network, University of Guelph, Ontario, Canada) for providing EBV for LP. This work was supported by grants from the Dairy Cattle Genetics Research and Development (DairyGen) Council of CDN (Guelph, Ontario, Canada) and from
Agriculture and Agri-Food Canada (AAFC RBPI 1276; Ottawa, Ontario, Canada).

\section{REFERENCES}

Alain, K., N. A. Karrow, C. Thibault, J. St-Pierre, M. Lessard, and N. Bissonnette. 2009. Osteopontin: An early innate immune marker of Escherichia coli mastitis harbors genetic polymorphisms with possible links with resistance to mastitis. BMC Genomics 10:444-461.

Appuhamy, J. A., B. G. Cassell, C. D. Dechow, and J. B. Cole. 2007. Phenotypic relationships of common health disorders in dairy cows to lactation persistency estimated from daily milk weights. J. Dairy Sci. 90:4424-4434.

Ashkar, S., G. F. Weber, V. Panoutsakopoulou, M. E. Sanchirico, M. Jansson, S. Zawaideh, S. R. Rittling, D. T. Denhardt, M. J. Glimcher, and H. Cantor. 2000. Eta-1 (osteopontin): An early component of type-1 (cell-mediated) immunity. Science 287:860-864.

Baik, M. G., M. J. Lee, and Y. J. Choi. 1998. Gene expression during involution of mammary gland. Int. J. Mol. Med. 2:39-44. (review).

Bartels, S. J., C. G. Spruijt, A. B. Brinkman, P. W. Jansen, M. Vermeulen, and H. G. Stunnenberg. 2011. A SILAC-based screen for Methyl-CpG binding proteins identifies RBP-J as a DNA methylation and sequence-specific binding protein. PLoS One 6:e25884.

Boettcher, P. 2005. Breeding for improvement of functional traits in dairy cattle. Ital. J. Anim. Sci. 4(3s):7-16.

Canadian Dairy Network. 2004. Genetic Selection for Persistency. Canadian Dairy Network, Guelph, Ontario, Canada.

Capuco, A. V., S. E. Ellis, S. A. Hale, E. Long, R. A. Erdman, X. Zhao, and M. J. Paape. 2003. Lactation persistency: Insights from mammary cell proliferation studies. J. Anim. Sci. 81(Suppl. 3):18-31.

Cole, J. B., and P. M. VanRaden. 2006. Genetic evaluation and best prediction of lactation persistency. J. Dairy Sci. 89:2722-2728.

Denhardt, D. T., and M. Noda. 1998. Osteopontin expression and function: Role in bone remodeling. J. Cell. Biochem. Suppl. 3031:92-102.

Denhardt, D. T., M. Noda, A. W. O'Regan, D. Pavlin, and J. S. Berman. 2001. Osteopontin as a means to cope with environmental insults: Regulation of inflammation, tissue remodeling, and cell survival. J. Clin. Invest. 107:1055-1061.

Do, D. N., N. Bissonnette, P. Lacasse, F. Miglior, M. Sargolzaei, X. Zhao, and E. M. Ibeagha-Awemu. 2017. Genome-wide association analysis and pathways enrichment for lactation persistency in Canadian Holstein cattle. J. Dairy Sci. 100:1955-1970.

Dudemaine, P. L., C. Thibault, K. Alain, and N. Bissonnette. 2014. Genetic variations in the SPP1 promoter affect gene expression and the level of osteopontin secretion into bovine milk. Anim. Genet. 45:629-640.

Gowda, C. S., C. Song, Y. Ding, M. Kapadia, and S. Dovat. 2016 Protein signaling and regulation of gene transcription in leukemia: Role of the Casein Kinase II-Ikaros axis. J. Investig. Med. 64:735-739. 
Herve, L., H. Quesnel, V. Lollivier, and M. Boutinaud. 2016. Regulation of cell number in the mammary gland by controlling the exfoliation process in milk in ruminants. J. Dairy Sci. 99:854-863.

Jakobsen, J. H., R. Rekaya, J. Jensen, D. A. Sorensen, P. Madsen, D. Gianola, L. G. Christensen, and J. Pedersen. 2003. Bayesian estimates of covariance components between lactation curve parameters and disease liability in Danish Holstein cows. J. Dairy Sci. 86:3000-3007.

Jamrozik, J. G. Jansen, L. Schaeffer, and Z. Liu. 1998. Analysis of persistency of lactation calculated from a random regression test day model. Interbull Bull. 17:64-69.

Jamrozik, J., L. R. Schaeffer, and G. B. Jansen. 2000. Approximate accuracies of prediction from random regression models. Livest. Prod. Sci. 66:85-92.

Konno, S., J. A. Eckman, B. Plunkett, X. Li, J. S. Berman, J. Schroeder, and S. K. Huang. 2006. Interleukin-10 and Th2 cytokines differentially regulate osteopontin expression in human monocytes and dendritic cells. J. Interferon Cytokine Res. 26:562-567.

Leyva-Baca, I., F. Schenkel, J. Martin, and N. A. Karrow. 2008. Polymorphisms in the $5^{\prime}$ upstream region of the CXCR1 chemokine receptor gene, and their association with somatic cell score in Holstein cattle in Canada. J. Dairy Sci. 91:407-417.

Liefke, R., F. Oswald, C. Alvarado, D. Ferres-Marco, G. Mittler, P. Rodriguez, M. Dominguez, and T. Borggrefe. 2010. Histone demethylase KDM5A is an integral part of the core Notch-RBP-J repressor complex. Genes Dev. 24:590-601.

Muir, B. L., J. Fatehi, and L. R. Schaeffer. 2004. Genetic relationships between persistency and reproductive performance in first-lactation Canadian Holsteins. J. Dairy Sci. 87:3029-3037.

Nayeri, S., M. Sargolzaei, M. K. Abo-Ismail, S. Miller, F. Schenkel, S. S. Moore, and P. Stothard. 2017. Genome-wide association study for lactation persistency, female fertility, longevity, and lifetime profit index traits in Holstein dairy cattle. J. Dairy Sci. 100:12461258.

Norgaard, J. V., P. K. Theil, M. T. Sorensen, and K. Sejrsen. 2008. Cellular mechanisms in regulating mammary cell turnover during lactation and dry period in dairy cows. J. Dairy Sci. 91:2319-2327.

O'Brien, J., H. Martinson, C. Durand-Rougely, and P. Schedin. 2012. Macrophages are crucial for epithelial cell death and adipocyte repopulation during mammary gland involution. Development 139:269-275.
Prokesch, A., A. Smorlesi, J. Perugini, M. Manieri, P. Ciarmela, E. Mondini, Z. Trajanoski, K. Kristiansen, A. Giordano, J. G. Bogner-Strauss, and S. Cinti. 2014. Molecular aspects of adipoepithelial transdifferentiation in mouse mammary gland. Stem Cells $32: 2756-2766$

Schwanbeck, R. 2015. The role of epigenetic mechanisms in Notch signaling during development. J. Cell. Physiol. 230:969-981.

Sodek, J., A. P. Batista Da Silva, and R. Zohar. 2006. Osteopontin and mucosal protection. J. Dent. Res. 85:404-415.

Sorensen, M. T., J. V. Norgaard, P. K. Theil, M. Vestergaard, and K. Sejrsen. 2006. Cell turnover and activity in mammary tissue during lactation and the dry period in dairy cows. J. Dairy Sci. 89:4632-4639.

Stefanon, B., M. Colitti, G. Gabai, C. H. Knight, and C. J. Wilde. 2002. Mammary apoptosis and lactation persistency in dairy animals. J. Dairy Res. 69:37-52.

Walker, D. 2014. Milk 2020: Genomic Profiling as a Prediction Tool for Future Milk Production. Growing Forward II program, Fredericton, New Brunswick, Canada.

Walsh, S. W., E. J. Williams, and A. C. Evans. 2011. A review of the causes of poor fertility in high milk producing dairy cows. Anim. Reprod. Sci. 123:127-138.

Wang, H., C. Song, Y. Ding, X. Pan, Z. Ge, B. H. Tan, C. Gowda, M. Sachdev, S. Muthusami, H. Ouyang, L. Lai, O. L. Francis, C. L. Morris, H. Abdel-Azim, G. Dorsam, M. Xiang, K. J. Payne, and S. Dovat. 2016. Transcriptional regulation of JARID1B/KDM5B histone demethylase by Ikaros, histone deacetylase 1 (HDAC1), and casein kinase 2 (CK2) in B-cell acute lymphoblastic leukemia. J. Biol. Chem. 291:4004-4018.

Wang, K. X., and D. T. Denhardt. 2008. Osteopontin: Role in immune regulation and stress responses. Cytokine Growth Factor Rev. 19:333-345.

Yart, L., L. Finot, V. Lollivier, and F. Dessauge. 2013. Oestradiol enhances apoptosis in bovine mammary epithelial cells in vitro. J. Dairy Res. 80:113-121.

Yart, L., V. Lollivier, P. G. Marnet, and F. Dessauge. 2014. Role of ovarian secretions in mammary gland development and function in ruminants. Animal 8:72-85.

Zeng, Z. B., T. Wang, and W. Zou. 2005. Modeling quantitative trait loci and interpretation of models. Genetics 169:1711-1725. 